Theorien gern mit der Wirklichkeit selbst verwechselt werden). In diesem Sinne hat sich der Überblicksradius der Parteienforschung seit 1989 für Detterbeck kaum verändert. Europa endet in diesem Buch offenbar auch heute im Bayerischen Wald und in Österreich. Verglichen mit der geographischen Wahrnehmungsgrenze der bundesdeutschen Parteienforschung des Jahres 1989 wagt Detterbeck immerhin eine Neuerung: Die Elbe weicht im Lehrbuch der Oder. Selbst wenn das Auslassen der Einschränkung „in westlichen Demokratien“ im Buchtitel lediglich einen Flüchtigkeitsfehler darstellen sollte, hätten im Text zumindest einige Parteien und Parteiensysteme jener ehemals kommunistisch regierten Länder Erwähnung finden müssen, die seit mehreren Jahren EU-Mitglieder sind. Recht viel könnte man daraus etwa über solche klassischen Themen der komparativen Parteienforschung lernen wie Cleavages, Entstehung und Organisation von Parteien sowie institutionelle Stabilität junger Parteiensysteme.

Selbst der bescheidene Anspruch aus dem Vorwort, einen Überblick über „die Parteienforschung" zu bieten, wird auch deshalb nicht eingelöst, weil Parteien in autoritären Systemen darin nicht einmal angesprochen werden. Unerwähnt bleiben sie ungeachtet der systemischen „Vorherrschaft“ des Autoritarismus in der gegenwärtigen Welt, der zunehmenden Bedeutung der Parteien in diesem Herrschaftssystem und des durchaus ausgiebigen Forschungsstandes.

Man könnte abschließend auch mit Hinblick auf die Zielgruppe des Lehrbuchs beklagen, dass sich Detterbeck ausschließlich an harmoniesüchtige Adepten des Fachs wendet beziehungsweise solche zu erziehen trachtet. Diese Kritik wäre aber nur dann gerecht, wenn in der deutschen Politikwissenschaft die besagte hegelianische Tradition nicht seit Jahrzehnten vorherrschend und die hier skizzierte Narration selbst für wissenschaftliche Qualifizierungsarbeiten nicht längst typisch geworden wäre. Also doch das perfekte Lehrbuch!

Jerzy Maćków

\title{
Parteientheorie und direkte Demokratie: gelungene Verknüpfung, übertriebene Einordnung der Befunde
}

Hornig, Eike-Christian: Die Parteiendominanz direkter Demokratie in Westeuropa, Nomos Verlagsgesellschaft, Baden-Baden 2011, 344 Seiten, € 59,-.

Eike-Christian Hornigs Grundidee ist es zwei Theorien zu verbinden, nämlich jene der direkten Demokratie und die Parteientheorie. Bei ersterer geht der Verfasser von Sabine Jungs „Logik der direkten Demokratie“ (2001) aus, für letztere knüpft er an den Parteiendominanzansatz von Richard S. Katz und Rudolf Wildenmann in der Weiterentwicklung durch Heidrun Abromeit an. Jungs Frage nach der Kompatibilität von direktdemokratischen Verfahren und repräsentativen Systemen bezieht Hornig also auf die Rolle der Parteien: Werden diese durch direkte Demokratie geschwächt, wie es lange ausgemacht schien, oder gilt umgekehrt, dass die Parteien die direkte Demokratie als neue Arena benutzen und dadurch an Stärke gewinnen, wie es in neueren Arbeiten vertreten wird? Seine Grundannahme, dass „Parteien auch die direktdemokratischen Prozesse in ,ihrem“ jeweiligen politischen System dominieren“ (S. 28) will Hornig für Dänemark, Frankreich, Großbritannien, die Nieder- 
lande, Italien, Schweden, die Schweiz, Österreich und Norwegen empirisch untersuchen. Andere in Betracht kommende Länder werden „vernachlässigt“, wobei das Fehlen Irlands nach des Verfassers eigener Einsicht eine „Leerstelle“ hinterlässt; die Begründung, dieses Land habe ,aufgrund seiner umfangreichen Praxis nicht mit berücksichtigt werden" können (S. 30), scheint auf dem Kopf zu stehen; näher liegt der Schluss, dass es deshalb unbedingt hätte einbezogen werden müssen. Auch Deutschland wird nicht behandelt, weil Hornig sich auf die nationale Ebene beschränkt.

Um für jene neun Länder den Grad des Parteieneinflusses zu bestimmen, entwickelt Hornig ein aufwendiges Untersuchungsdesign. Den drei Phasen eines idealtypischen direktdemokratischen Prozesses entsprechend wird erstens gefragt: Wer leitet eine Abstimmung ein (Kompetenz für Auslösung und Urheberschaft)? Zweitens: Welchen Einfluss haben Parteien im Abstimmungsprozess, beziehungsweise „wie oft weichen die Abstimmungsergebnisse von dem ab, was die Parteien eigentlich wollten" (S. 61)? Drittens: Wie verbindlich ist die direktdemokratisch getroffene Entscheidung (Bestand und Abänderbarkeit)? Diese Bewertungsmaßstäbe werden dann in Auseinandersetzung mit der Literatur ausdifferenziert und operationalisiert, indem der jeweils zu bestimmende Parteieneinfluss auf einer Skala von 0 bis 3 Punkten angesetzt wird, die Hornig noch durch Reduzierung von halben oder gar Viertelpunkten verfeinert.

Das so entwickelte Instrumentarium wird auf die Untersuchungsländer in angemessen gestufter Form angewandt: eingehende Abhandlungen von über 50 Seiten für Italien, mit dem Hornig durch einen Studienaufenthalt besonders vertraut ist, und die Schweiz; mittlere Darstellungen für Dänemark und Frankreich sowie knappe Skizzen von unter zehn Seiten für Großbritannien und die Niederlande. Diese Länderstudien können hier nicht im Einzelnen gewürdigt werden. Sie sind, pauschal gesagt, sehr informativ, ihre Ergebnisse wirken plausibel, und man liest sie mit Gewinn. Hornig hat damit eine respektable Leistung erbracht.

Allerdings gibt es hier auch Anlass zu Kritik: So begeht der Verfasser den klassischen Fehler, mit „weichen “ Daten „hart" zu rechnen. Die Punkteskala ist bei aller Verfeinerung doch angesichts der realen Komplexität der entsprechenden sozialen Vorgänge eine zu rigorose Vereinfachung, um überhaupt rechnen zu können. Wenn Hornig etwa beim Verfassungsreferendum in Frankreich erkennt, dass der dortige Auslösungsmodus in seiner Bewertungstabelle gar nicht vorgesehen ist, und deshalb eine „individuelle Bewertung" vornimmt (S. 106), überzeugt ein Schlusswert von 1,16 Punkten (S. 110) kaum, erst recht nicht die Absetzung von dem präsidentiellen Verfahren ebendort, das nur 1,04 Punkte (S. 131) bekommt. Solche Rechenergebnisse mit zwei Dezimalen sind pseudogenau. Umgekehrt gibt Hornig bei der Verfassungsinitiative in der Schweiz - die 14 Seiten liest man hierzu mit besonderem Interesse, nachdem „direkte Demokratie“ in den anderen acht Ländern immer Referendumsdemokratie bedeutet, bei der ja der Parteieneinfluss offenkundig ist - für einen Sachverhalt zwei einander widersprechende Interpretationen: Dass insgesamt 16 beziehungsweise 18 von 100 Vorlagen allein von Parteien angestoßen wurden, soll einmal „eine bedeutende Rolle“ der Parteien belegen (S. 250), während ein andermal ihre Rolle zu denselben Fakten als ,nahezu marginal“ bezeichnet wird (S. 304).

Hornigs Ergebnisse lauten: „Direkte Demokratie wird in Westeuropa größtenteils von den Parteien bestimmt" (S. 330). Es lasse sich eine ausgeprägte bis mittlere Parteiendominanz zeigen. Die Ergebnisse von Volksabstimmungen stimmten durchschnittlich zu 83,6 Prozent mit „den repräsentativen Kräfteverhältnissen“ überein (S. 314). Somit erweise sich 
direkte Demokratie in der Masse der Abstimmungen „als ungeeignet als Instrument gegen die Dominanz der Parteien“ (S. 323). Gegen Jung und ihre Systemtyp-Erklärung pointiert Hornig, „dass die wahre Logik direkter Demokratie jene der repräsentativen Sphäre (mit nur wenigen Ausflüchten) ist“ (S. 335).

Leider schmälert der Verfasser dieser bei Ralf Kleinfeld in Osnabrück entstandenen Dissertation seine Verdienste, wenn er formuliert, „das Bild, das von direkter Demokratie allenthalben verbreitet wird, (müsse) korrigiert werden. Volksabstimmungen sind nur sehr selten ein Korrektiv der repräsentativen Akteure“ (S. 330). Oder gar, seine Ergebnisse lieferten „einen bislang fehlenden Realismus für den Umgang mit direkter Demokratie“ (S. 335).

Tilman Evers hat schon vor Jahrzehnten in einem eher geisteswissenschaftlichen Zugriff bezweifelt, dass direkte Demokratie „ein wirksames Mittel gegen den Parteien-Absolutismus wäre“ 1 . Volksentscheide könnten sich „nur punktuell und begrenzt unterscheiden von dem, was heute im parlamentarischen Verfahren an Resultaten durchsetzbar ist. Dieser geringe Unterschied kann im Einzelfall wichtig sein “2. In seinem letzten Satz schwang allerdings ein bisschen Hoffnung mit, während Hornig mit der Rede von „Störfaktor“ und „Denkzettel-Abstimmungen“ schon sprachlich die Parteienperspektive verinnerlicht hat.

Otmar Jung

1 Tilman Evers, Mehr Demokratie durch Volksentscheid?, in: Kritische Justiz, 19. Jg. (1986), H. 4, S. $423-433$, S. 429.

2 Ders., Zivilgesellschaft und direkte Demokratie in gesamtdeutscher Verfassung, in: Staat und Recht, 39. Jg. (1990), H. 4, S. 931 - 945, S. 942.

\section{Zweite Kammern: gehaltvolle Analysen, editorische und redaktionelle Mängel}

\section{Leunig, Sven (Hrsg.): Handbuch Föderale Zweite Kammern, Verlag Barbara Budrich, Opladen} I Farmington Hills 2009, 313 Seiten, € 48,-.

Riescher, Gisela, Sabine Ruß und Christoph M. Haas: Zweite Kammern, 2. überarbeitete und erweiterte Auflage, Oldenbourg Wissenschaftsverlag GmbH, München 2010, 576 Seiten, $€ 49,80$.

Das Schattendasein, das viele Zweite Kammern sowohl in der öffentlichen als auch in der politikwissenschaftlichen Wahrnehmung führen, entspricht nicht zwangsläufig ihrer tatsächlichen Bedeutung im jeweiligen politischen System. Diese Einsicht gewinnt man aus beiden Handbüchern. Dennoch unterscheiden sich beide Bände deutlich - sowohl in ihrem Anspruch als auch in der Qualität von Darstellung und Analyse.

Der von Sven Leunig herausgegebene Band soll eine Forschungslücke schließen. Es handelt sich um die erste Darstellung, in der alle föderalen Zweiten Kammern vergleichend betrachtet werden. Von diesem sehr breiten Ansatz - immerhin werden die 20 Zweiten Kammern aller Bundesstaaten weltweit untersucht, einschließlich eines Exkurses auf den im Jahr 2000 abgeschafften Senat Venezuelas - verspricht sich der Herausgeber auch eine 